\title{
Cardiovascular and metabolic risk profile in young people at familial risk of depression
}

\author{
Zola N. Mannie, Clare Williams, Jonathan Diesch, Andrew Steptoe, Paul Leeson
} and Philip J. Cowen

\section{Background}

Depression is associated with increased risk of several general medical conditions, including diabetes and cardiovascular disease. The nature of the association is complex and may involve bidirectional causation or a common pathophysiology.
Aims
To determine whether young people without depression but at increased familial risk have altered metabolic and blood pressure markers relative to matched controls.

\section{Method}
We studied young people $(n=85)$, who had a parent with depression but no personal history of depressive illness ( $\mathrm{FH}+$ ) and healthy controls $(n=69)$. Cardiovascular risk profile was assessed by a fasting blood sample to measure insulin, glucose, lipids and high-sensitivity C-reactive protein (CRP) and blood pressure was measured centrally and peripherally. Arterial stiffness and waking cortisol concentration were also measured.

\section{Results}

Compared with controls, the $\mathrm{FH}+$ group demonstrated increased peripheral and central systolic blood pressure, increased arterial stiffness and diminished insulin sensitivity but they did not differ from controls in measures of lipids, CRP or waking cortisol.

\section{Conclusions}

Our data suggest that young people at increased familial risk of depression show evidence of altered cardiovascular risk profile in young adulthood even in the absence of depressive symptoms. It is possible therefore that vulnerability to conditions such as hypertension and diabetes may precede the onset of major depression and may share common risk factors.

\section{Declaration of interest}

P.J.C. is a paid member of a scientific advisory board for Lundbeck.
There are well-established links between major depression and general medical conditions such as diabetes and cardiovascular disease. For example, a meta-analysis of 11 epidemiological studies indicated that the relative risk of future coronary heart disease in people with depressive symptomatology was 1.81 (95\% CI 1.53-2.15). ${ }^{1}$ In a Finnish study of 2500 young men, those with depressive symptomatology had significantly elevated risk of manifesting insulin resistance (odds ratio $(\mathrm{OR})=3.15,95 \%$ CI $1.48-6.68),{ }^{2}$ which is itself a risk factor for the development of type 2 diabetes and cardiovascular disease. $^{3,}$ To some extent this association might reflect the consequences of depression and its treatment. For example, depression can be associated with diminished self-care and activity and many psychotropic medications cause excessive weight gain. However, it is also possible that depression and some medical conditions could share common aetiological factors. ${ }^{4}$

If the latter is the case it might be expected that markers of potential general medical comorbidity would be present at the onset of depression or even before its development in high-risk individuals. For instance, a recent study reported that decreased cardiovascular fitness in male conscripts predicted future serious depressive disorder. ${ }^{5}$ In a previous investigation we found elevated waking salivary cortisol levels in young people at increased familial risk of depression through virtue of having a parent with depression, ${ }^{6}$ and increased cortisol secretion has been linked to the development of hypertension, diabetes and obesity. ${ }^{7}$ In the present study therefore, we examined a similar group of at-risk young people to assess whether they exhibited differences from controls in a variety of measures linked to metabolic and cardiovascular disease.

\section{Method}

\section{Participants and psychosocial measures}

We recruited 85 young people ( 53 women, 32 men) with a mean age of 18.9 years (s.d. $=1.0$, range 16-20) who had never personally had depression but who had a biological parent with a history of major depression $(\mathrm{FH}+)$. Potential participants were assessed with the Structured Clinical Interview for DSM-IV Axis I Disorders (SCID-I) ${ }^{8}$ to exclude a personal current or previous history of major depression. The presence of major depression in a parent was assessed by the family history method using the participant as an informant. ${ }^{9}$ The criteria used included description of the symptoms of major depression together with the prescription of specific antidepressant treatment, either psychotherapy or medication. This was followed up by direct verification from the affected parent (either by telephone or in writing); where parental history could not be verified, participants were excluded. A history of bipolar disorder in a parent was an exclusion criterion. Of the affected parents, 79 had been treated with antidepressant medication and 68 had been referred for specialist assessment. In total, 71 had experienced more than one episode of depression and 11 had received in-patient treatment for depression at some time. We also recruited 69 controls $(42$ women and 27 men) with a mean age of 19.1 years (s.d. $=0.8$ years, range 16-20) who were determined by the same instruments to have no current or past history of major depression and no history of depression in a biological parent or other first-degree relative.

Participants were assessed for current mood and anxiety symptoms with the Hospital Anxiety and Depression Scale 
(HADS $),{ }^{10}$ and the Perceived Stress Scale (PSS) ${ }^{11}$ was used to give a measure of subjective stress over the past month. ${ }^{7}$ Adverse life events and the impact of these events on emotional well-being were assessed with the Life Events Rating Scale (LERS), ${ }^{12}$ which assesses adverse events at two time points; first, at a distal time point that includes childhood adversity and second, events experienced in the past year. We assessed the quality of perceived parenting style for the first 16 years of life with the Parental Bonding Instrument (PBI), ${ }^{13}$ obtaining both maternal and paternal PBI scores. Physical activity was assessed with the selfrated International Physical Activity Questionnaire (IPAQ), ${ }^{14}$ which measures the number of minutes per week spent in physical activities. Parental history of diabetes and cardiovascular disease was sought from each participant. All participants gave full informed consent to the study, which was approved by the local ethics committee.

\section{Metabolic and cardiovascular measures}

Participants were asked to fast from midnight and present to the unit the next day at approximately $08.30 \mathrm{~h}$ for fasting blood sampling of glucose and insulin. We also sampled for lipid profile as well as high-sensitivity C-reactive protein (CRP), a general measure of inflammation. ${ }^{15}$ We measured body weight and height to calculate body mass index (BMI) and also waist and hip circumference for waist/hip ratio.

Blood samples were drawn, centrifuged and separated within $30 \mathrm{~min}$, then stored at $-20^{\circ} \mathrm{C}$ for later analysis. Fasting blood glucose, insulin, lipids and CRP were measured at the Oxford Radcliffe Hospital Biochemistry Laboratory using routine validated clinical biochemistry assays masked to diagnostic status. Homeostatic model assessment to determine insulin sensitivity was calculated with the HOMA2 computer model (HOMA calculator, version 2.2; Diabetes Trials Unit, Oxford Centre for Diabetes, Endocrinology and Medicine, Oxford, UK, www.dtu.ox. ac.uk/homacalculator/). ${ }^{16}$

\section{Peripheral and central blood pressure and aortic stiffness}

Peripheral blood pressure readings were obtained following 15 min supine rest using a calibrated oscillometric device. Radial artery waveform was recorded by applanation tonometry of the radial pulse to generate an ascending aortic waveform and central blood pressure derived based on a mathematical transfer function. ${ }^{17}$ Aortic stiffness was assessed non-invasively by measuring carotid-femoral pulse wave velocity as previously described. ${ }^{18}$

\section{Salivary cortisol sampling}

Participants were asked to provide waking saliva samples with a salivette device (Sarstedt, Leicester, UK, www.sarstedt.com) for cortisol measurement. They performed sampling at home on a work day. Participants began sampling as soon as they woke up and remained resting in bed and fasting for a further $30 \mathrm{~min}$ during which they took two further saliva samples at 15-minute intervals, as previously described. ${ }^{6}$ Plasma cortisol was measured by direct double-antibody radioimmunoassay utilising ${ }^{125} \mathrm{I}$ labelled cortisol. The inter- and intra-assay coefficients of variation were $6 \%$ and $4.9 \%$ respectively and the limit of detection was $0.3 \mu \mathrm{g} / \mathrm{ml}$.

\section{Statistical analysis}

All data were analysed with SPSS v20 for Windows. Analyses were conducted using unpaired $t$-tests (two-tailed) or analysis of variance (ANOVA) with 'group' (FH+ participants $v$. controls) and 'gender' as between-participant factors. The glucose, insulin and homeostasis model of assessment - insulin resistance index (HOMA-IR) - data were skewed, and to normalise the distribution in preparation for analysis, logarithmic transformation of the data was performed as previously recommended. ${ }^{16}$ Where necessary, covariates were added (ANCOVA) to control for factors such as BMI and family history of medical illness. Categorical data were analysed with the $\chi^{2}$ test and correlations carried out using Pearson's product moment.

\section{Results}

\section{Psychosocial measures and health-related behaviours}

The groups were well-matched for age and gender and had similar scores for current mood and anxiety and perceived stress. There were no significant differences in reported adverse life events, either recent or lifetime. The FH+ group and controls also scored similarly in measures of parental attachment in the first 16 years of life (Table 1). There were no group differences in BMI, waist/hip ratio, smoking, alcohol consumption and physical activities of all intensities (Table 2). However, of the 85 individuals in the $\mathrm{FH}+$ group, 6 reported a parent with diabetes compared with none from the 69 controls $\left(\chi^{2}=5.07, P=0.02\right)$. Only one of the FH+ group and two of the control group reported a parental history of cardiovascular disease $\left(\chi^{2}=0.59, P=0.44\right)$.

\section{Glucose regulation, lipids, CRP and cortisol}

Fasting glucose levels were similar between the two groups $(F=0.23$, d.f. $=1,142, P=0.63)$; however, the $\mathrm{FH}+$ group showed increased insulin levels $(F=9.9$, d.f. $=1,139, P=0.002)$ and also increased insulin resistance on the HOMA-IR $(F=7.6$, d.f. $=1,130, P=0.007$ ) (Table 3). The ANOVAs for both glucose and the HOMA-IR showed significant main effects of gender (glucose: $F=4.17$, d.f. $=1,42, P=0.043$; HOMA-IR: $F=4.50$, d.f. $=1,130, P=0.036$ ) where men had mildly greater glucose levels and lower HOMA-IR scores than women (data not shown); however, there were no significant group $\times$ gender interactions (all $P$-values $>0.1$ ). The effect of $\mathrm{FH}+$ status on insulin levels and HOMA-IR remained significant when controlling for parental history of diabetes $(F=10.66$, d.f. $=1,138, P=0.001$, and $F=8.37$, d.f. $=1,129, P=0.004)$.

None of the lipid measures distinguished the FH+ group from the controls and there were no significant interactions between group and gender for any of the lipid measures (all $P$-values $>0.1$ ). Similarly there was no difference in CRP values between participants in the $\mathrm{FH}+$ group and controls, and no significant gender $\times$ group interactions (all $P$-values $>0.1$ ) (Table 3 ).

Assayable salivary cortisol was obtained from 147 participants ( 81 in the $\mathrm{FH}+$ group and 66 in the control group). Participants with initial waking salivary cortisol values more than 3 standard deviations from the mean (one from each group) were excluded. Area under the curve (AUC, without subtraction for baseline secretion) was computed and no significant difference was found between the $\mathrm{FH}+$ and control group in salivary cortisol secretion $(F=1.88$, d.f. $=1,141, \quad P=0.172) \quad$ (Table 3$)$. There was no significant main effect of gender or gender $\times$ group interaction (all $P$-values $>0.1$ ).

\section{Cardiovascular measures}

The ANOVA of peripheral systolic blood pressure showed a main effect of group $(F=6.01$, d.f. $=1,142, P=0.015)$; there was also a main effect of gender $(F=10.2$, d.f. $=1,42, P=0.002)$ but no group gender interaction $(F=0.16$, d.f. $=1,142, P=0.69)$. Mean peripheral 
Table 1 Demographic and other psychosocial characteristics of participants

\begin{tabular}{|c|c|c|c|}
\hline & $\begin{array}{l}\mathrm{FH}+\text { group }^{\mathrm{a}} \\
\quad(n=85)\end{array}$ & $\begin{array}{l}\text { Control group } \\
\quad(n=69)\end{array}$ & $P$ \\
\hline Age, years: mean (s.e.m.) & $18.9(0.1)$ & $19.1(0.1)$ & 0.20 \\
\hline Gender, male/female, $n$ & $32 / 53$ & $27 / 42$ & 0.85 \\
\hline \multicolumn{4}{|c|}{ Hospital Anxiety and Depression Scale, mean (s.e.m.) } \\
\hline Depression subscale & $1.8(0.3)$ & $1.4(0.2)$ & 0.15 \\
\hline Anxiety subscale & $4.3(0.4)$ & $4.0(0.3)$ & 0.50 \\
\hline Perceived Stress Scale, mean (s.e.m.) & $13.3(0.6)$ & $13.4(0.7)$ & 0.89 \\
\hline \multicolumn{4}{|l|}{ Life Events Rating Scale, ${ }^{\mathrm{b}}$ mean (s.e.m.) } \\
\hline Recent & $0.9(0.1)$ & $0.7(0.1)$ & 0.13 \\
\hline Lifetime & $0.5(0.1)$ & $0.4(0.1)$ & 0.38 \\
\hline \multicolumn{4}{|c|}{ Maternal - Parental Bonding Instrument, ${ }^{\mathrm{C}}$ mean (s.e.m.) } \\
\hline Care & $30.0(0.7)$ & $30.4(0.8)$ & 0.71 \\
\hline Overprotect & $10.5(0.8)$ & $10.3(0.8)$ & 0.84 \\
\hline \multicolumn{4}{|c|}{ Paternal - Parental Bonding Instrument, ${ }^{\mathrm{c}}$ mean (s.e.m.) } \\
\hline Care & $27.7(0.8)$ & $26.4(0.9)$ & 0.81 \\
\hline Overprotect & $7.9(0.7)$ & $7.8(0.8)$ & 0.88 \\
\hline $\begin{array}{l}\text { a. Participants with a parent with depression } \\
\text { b. Life Events Rating Scale: recent, in the pas } \\
\text { c. Parental Bonding Instrument: care, lack of }\end{array}$ & & & \\
\hline
\end{tabular}

\begin{tabular}{|c|c|c|c|}
\hline & \multicolumn{2}{|c|}{ Mean (s.e.m.) } & \multirow[b]{2}{*}{$P$} \\
\hline & $\mathrm{FH}+$ group $^{\mathrm{a}}$ & Control group & \\
\hline Alcohol, units/week & $8.9(0.9)$ & $10.1(1.2)$ & 0.40 \\
\hline Smokers/non-smokers & $16 / 69$ & $13 / 56$ & 1.00 \\
\hline Body mass index & $23.0(0.4)$ & $22.6(0.3)$ & 0.48 \\
\hline Waist/hip ratio & $0.75(0.01)$ & $0.76(0.01)$ & 0.84 \\
\hline \multicolumn{4}{|c|}{ Physical activity (MET mins/week) ${ }^{b}$} \\
\hline Walking & 891 (89) & 1001 (103) & 0.42 \\
\hline Moderate & $585(87)$ & $522(61)$ & 0.56 \\
\hline Vigorous & $1168(164)$ & $1548(207)$ & 0.15 \\
\hline Total & 2734 (215) & 3297 (387) & 0.14 \\
\hline
\end{tabular}

\begin{tabular}{|c|c|c|c|}
\hline & \multicolumn{2}{|c|}{ Mean (s.e.m.) } & \multirow[b]{2}{*}{$P$} \\
\hline & $\mathrm{FH}+$ group $^{\mathrm{a}}$ & Control group & \\
\hline Glucose, $\mathrm{mmol} / \mathrm{l}$ & $4.53(0.04)$ & $4.56(0.06)$ & $0.63^{\mathrm{b}}$ \\
\hline Insulin, pmol/l & $67.7(5.1)$ & $52.2(3.8)$ & $0.002^{b}$ \\
\hline Homeostatic model assessment - insulin resistance index & $1.14(0.07)$ & $0.93(0.06)$ & $0.007^{b}$ \\
\hline Total cholesterol, mmol/l & $4.2(0.1)$ & $4.1(0.1)$ & 0.35 \\
\hline High-density lipoprotein, mmol/l & $1.3(0.1)$ & $1.4(0.1)$ & 0.54 \\
\hline Triglycerides, mmol/l & $1.0(0.06)$ & $0.94(0.05)$ & 0.43 \\
\hline C-reactive protein, $\mu \mathrm{g} / \mathrm{dl}$ & $2.4(0.6)$ & $2.2(0.57)$ & 0.73 \\
\hline $\mathrm{CORT}^{\mathrm{C}}$ & $228(12)$ & $206(12)$ & 0.17 \\
\hline
\end{tabular}

systolic blood pressure was higher in the $\mathrm{FH}+$ group than controls (Table 4) and higher in men than in women $(116.5$ (s.d.=1.4) v. 111.3 (s.d. $=1.0), P=0.002$ ). Central systolic blood pressure was similarly higher in the $\mathrm{FH}+$ group $(F=7.99$, d.f. $=1,142$, $P=0.005$ ) with no main or interactive effects of gender (all $P$-values $>0.1)$. There was a trend for peripheral diastolic blood pressure to be higher in the $\mathrm{FH}+$ group $(F=3.34$, d.f. $=1,142$, $P=0.07)$. There was also a main effect of gender $(F=24.53$, d.f. $=1,142, P=0.001)$ with peripheral diastolic blood pressure being higher in women (mean $62.3 \quad$ (s.d.=1.1) v. 68.7 (s.d. $=0.8), P=0.001)$. There was no interaction between group and gender $(F=0.055$, d.f. $=1,142, P=0.81)$. Central diastolic blood pressure did not differ between the $\mathrm{FH}+$ group and controls ( $F=2.32$, d.f. $=1,142, P=0.13)$. However, central diastolic blood pressure was higher in women than men $(F=25.81$, d.f. $=1,142$, $P=0.001$; men 62.8 (s.d.=1.1) v. 69.8 (s.d. $=0.9), P=0.001)$. 
Table 4 Cardiovascular measures

\begin{tabular}{|c|c|c|c|}
\hline & \multicolumn{2}{|c|}{ Mean (s.e.m.) } & \multirow[b]{2}{*}{$P$} \\
\hline & $\mathrm{FH}+$ group $^{\mathrm{a}}$ & Control group & \\
\hline \multicolumn{4}{|c|}{ Systolic blood pressure, $\mathrm{mmHg}$} \\
\hline Peripheral & $115.1(1.2)$ & $111.3(1.2)$ & 0.015 \\
\hline Central & $96.8(0.9)$ & $93.1(1.2)$ & 0.005 \\
\hline \multicolumn{4}{|c|}{ Diastolic blood pressure, $\mathrm{mmHg}$} \\
\hline Peripheral & $67.3(0.9)$ & $64.9(1.0)$ & 0.07 \\
\hline Central & $68.1(0.9)$ & $65.9(1.1)$ & 0.13 \\
\hline Pulse wave velocity, $\mathrm{m} / \mathrm{s}$ & $5.3(0.07)$ & $5.1(0.05)$ & 0.033 \\
\hline
\end{tabular}

Finally, pulse wave velocity was significantly higher in the FH+ group than controls $(F=4.62$, d.f. $=1,135, P=0.033)$ with no main or interactive effects of gender (all values $>0.1$ ) (Table 4). Controlling for family history of cardiovascular disease did not lead to any meaningful changes in the significance levels of the positive cardiovascular findings (data not shown).

\section{Correlations}

In all participants considered together, waist/hip ratio correlated positively and significantly with fasting insulin $(r=0.24$, $P=0.007)$ and peripheral systolic blood pressure $(r=0.18$, $P=0.041)$. The BMI correlated positively and significantly with fasting insulin $(r=0.28, \quad P=0.001)$, HOMA-IR $\quad(r=0.29$, $P=0.001)$, peripheral systolic blood pressure $(r=0.218$, $P=0.009)$, central systolic blood pressure $(r=0.25, P=0.002)$ and pulse wave velocity $(r=0.21, P=0.015)$. Including BMI as a covariate did not produce any meaningful changes in the statistical differences found between the FH+ group and controls in glucose regulation and cardiovascular measures. In the FH+ group considered alone there was no correlation between either CRP or cortisol AUC and fasting insulin levels or peripheral and central systolic blood pressure or pulse wave velocity. Finally, there was no correlation in this group between fasting insulin levels and any of the above cardiovascular measures.

\section{Discussion}

\section{Main findings}

The main finding of our study is that young people at increased familial risk of depression have increased systolic blood pressure and arterial stiffness and diminished insulin sensitivity relative to matched controls. These observations are of interest in view of the increased liability of patients with depression to experience diabetes and cardiovascular disease, including hypertension. ${ }^{4,7}$

The differences we saw between the $\mathrm{FH}+$ group and healthy controls in insulin sensitivity and systolic blood pressure are not apparently attributable to current health-related factors such as exercise, smoking, alcohol consumption or obesity. In addition, family history of general medical conditions, within the limitations of our ascertainment, does not seem to account for the differences in blood pressure and insulin sensitivity. However, clearly it would have been better to have questioned parents directly about their general medical health.

\section{Link between depression and diabetes}

It is well established that there is an association between depression and diabetes but the nature of the causal link is complex. The prevalence of depression is increased in patients with type 2 diabetes, whereas depression is also a risk factor for the subsequent development of diabetes. ${ }^{19,20}$
Depressive symptoms are associated with insulin resistance, ${ }^{2,21}$ which is itself a risk factor for diabetes. ${ }^{3}$ Our data suggest that a degree of insulin resistance is also present in young people without depression at familial risk of illness. The mechanisms that link depression with insulin resistance are not clear. It is possible, for example, that depression increases the prevalence of adverse health behaviours such as physical inactivity and obesity ${ }^{20}$ but this does not seem to be a factor in the present findings. In addition, biochemical changes associated with depression, for example elevated levels of cortisol and inflammatory cytokines, may be implicated in the development of insulin resistance. ${ }^{7,20}$ Our study, however, does not suggest that our $\mathrm{FH}+$ group have increased cortisol secretion as measured by the waking cortisol response. In addition, there was no difference between the $\mathrm{FH}+$ group and controls in levels of high-sensitivity CRP, a general measure of inflammation. ${ }^{15}$

\section{Link between depression and hypertension}

As with diabetes, it appears that depression predisposes to hypertension. For example, in a meta-analysis of nine studies (22367 participants), Meng et $a l^{22}$ found that after adjustment for confounders, significant depressive symptomatology increased the relative risk of developing hypertension (1.81, 95\% CI 1.09 1.86) and a further meta-analysis found an association between depression and subsequent stroke. ${ }^{23}$ Conversely, as noted above, diminished cardiovascular fitness in young people increased the risk of developing severe depression subsequently. ${ }^{5}$ In our study we found that young people with a family history of depression but who themselves were not depressed had a 3-4 mmHg higher peripheral systolic blood pressure, consistent with a change in cardiovascular risk profile, that pre-dates clinical symptoms of depression. This increase in blood pressure is also accompanied by a small $0.2 \mathrm{~m} / \mathrm{sec}$ higher pulse wave velocity and increases in central systolic blood pressure. Both of these measures provide information about central haemodynamics relevant to organ perfusion and in older individuals predict cardiovascular events independently of brachial measures. ${ }^{24}$

\section{Impact of childhood adversity}

Young people who have experienced a variety of social disadvantages as children (social isolation, low socioeconomic status, parental maltreatment) show elevated risk markers for cardiovascular disease, including increased blood pressure, abnormal lipid profile, high glycated haemoglobin (a measure of insulin resistance) and increased CRP, when examined in their 20s and 30 s. $^{25,26}$ These findings have some similarities to our own in terms of hypertension and insulin resistance but the mechanisms involved may be different because levels of childhood adversity did not apparently distinguish our two participant groups, at least as measured by life events and parental relationships. Indeed, Danese et al ${ }^{26,27}$ suggest that the raised CRP levels seen in their socially disadvantaged participants may be a consequence of childhood hardship and could be an important pathophysiological mechanism leading to the development of cardiovascular risk markers and cardiovascular disease. However, our findings suggest that increased familial risk of depression does not itself lead to increased levels of CRP despite also being associated with insulin resistance and increased blood pressure. However, it would have been better had we measured childhood maltreatment more directly.

\section{Use of elevated waking cortisol secretion as a marker}

As noted above, raised cortisol levels have also been implicated in the association between depression and diabetes, and depression 
and hypertension. ${ }^{7}$ Whereas in a previous study in $\mathrm{FH}+$ participants we found increased waking salivary cortisol secretion, ${ }^{7}$ such an effect was not apparent in the current $\mathrm{FH}+$ group. We are uncertain of the reason for this. Vreeburg et $a l^{28}$ also reported raised waking cortisol secretion in young people with a parental history of depression but only in those in whom the parental history was specifically verified during a structured interview with the relevant parent. The information we obtained from parents was less systematic, which could perhaps account for the lack of difference we found in cortisol secretion between the FH+ group and controls in the current study. It is also possible, of course, that elevated waking cortisol secretion is not a robust marker of familial predisposition to depression.

\section{Possible explanations for our findings}

At present the mechanism by which increased familial risk of depression is associated with altered insulin secretion and systolic blood pressure is unclear. Genetic factors are likely to explain part of the increased risk of familial depression but reliable identification of susceptibility genes has proved elusive. ${ }^{29}$ Nevertheless, it is possible that common genetic mechanisms might underlie both the risk of depression and cardiovascular disease, and a recent study in schizophrenia (which is also associated with an increased risk of cardiovascular disease) used a 'pleiotropyinformed' approach to identify a number of single nucleotide polymorphisms common to both disorders. ${ }^{30}$ It is also worth noting that, mechanistically, insulin resistance has been linked to depression in a functional imaging study of healthy adults in which higher levels of insulin resistance covaried with increased functional connectivity between ventral striatum and cingulate cortex, which in turned correlated with depressive symptoms. ${ }^{31}$ Finally, in a subset of the sample we found increased saliva levels of the mineralocorticoid, aldosterone (details available from the author on request), which is of interest in view of the role of this adrenal hormone in stress and blood pressure regulation. ${ }^{32}$ However, this finding was unexpected and requires replication.

In conclusion, our data suggest that young people with a parent with depression show changes in markers of insulin sensitivity and blood pressure regulation. This implies that the association between depression, diabetes and cardiovascular disease may pre-date the onset of clinical depression and supports the notion that depression and certain general medical conditions could share common pathophysiological mechanisms. Our data do not provide information on what these mechanisms might be; however, the current findings argue against an important role for either inflammation or cortisol hypersecretion in this particular group of at-risk individuals. Further work is needed to confirm these findings and to explore other potential neurobiological and social mechanisms.

Zola N. Mannie, MSC, PhD, Clare Williams, RMN, BSC, Psychopharmacology Research Unit, University Department of Psychiatry, Warneford Hospital, Oxford; Jonathan Diesch, Oxford Cardiovascular Clinical Research Facility, John Radliffe Hospital, Oxford; Andrew Steptoe, DSC, Institute of Epidemiology and Health Care University College London; Paul Leeson, PhD, FRCP, Oxford Cardiovascular Clinical Research Facility, John Radliffe Hospital, Oxford; Philip J. Cowen, MD, FRCPsych, Psychopharmacology Research Unit, University Department of Psychiatry, Warneford Hospital, Oxford, UK

Correspondence: Philip J. Cowen, Neurosciences Building, Warneford Hospital, Oxford OX3 7JX, UK. Email: phil.cowen@psych.ox.ac.uk

First received 27 Jan 2013, final revision 10 Apr 2013, accepted 17 Apr 2013

\section{Funding}

The study was supported by the Medical Research Council (G0900576).

\section{References}

1 Nicholson A, Kuper H, Hemingway $H$. Depression as an aetiologic and prognostic factor in coronary heart disease: a meta-analysis of 6362 events among 146538 participants in 54 observational studies. Eur Heart J 2006; 27: 2763-74.

2 Timonen M, Rajala U, Jokelainen J, Keinanen-Kiukaanniemi S, Meyer-Rochow VB, Rasanen P. Depressive symptoms and insulin resistance in young adult males: results from the Northern Finland 1966 birth cohort. Mol Psychiatry 2006; 11: 929-33.

3 Tabák AG, Jokela M, Akbaraly TN, Brunner EJ, Kivimäki M, Witte DR. Trajectories of glycaemia, insulin sensitivity and insulin secretion before diagnosis of type 2 diabetes: an analysis from the Whitehall II study. Lancet 2009; 373: 2215-21.

4 Farmer A, Korszun A, Owen MJ, Craddock N, Jones L, Jones I, et al. Medical disorders in people with recurrent depression. Br J Psychiatry 2008; 192: 351-5.

5 Åberg MAl, Waern M, Nyberg J, Pedersen NL, Bergh Y, Åberg ND, et al. Cardiovascular fitness in males at age 18 and risk of serious depression in adulthood: Swedish prospective population-based study. Br J Psychiatry 2012; 201: 352-9.

6 Mannie ZN, Harmer CJ, Cowen PJ. Increased waking salivary cortisol levels in young people at familial risk of depression. Am J Psychiatry 2007; 164: 617-21.

7 Brown ES, Varghese FP, McEwan BS. Association of depression with medical illness. Biol Psychiatry 2004; 55: 1-9.

8 First MB, Spitzer RL, Gibbon M, Williams JB. Structured Clinical Interview for DSM-IV Axis I Disorders. New York State Psychiatric Institute, 1995.

9 Andreason NC, Rice J, Endicott J. The family history approach to diagnosis. How useful is it? Arch Gen Psychiatry 1986; 43: 421-9.

10 Zigmond AS, Snaith RP. The Hospital Anxiety and Depression Scale. Acta Psychiatr Scand 1983; 67: 361-70.

11 Cohen S, Kamarck T, Mermelstein R. A global measure of perceived stress $J$ Health Soc Behav 1983; 24: 386-96.

12 Goodyer IM, Herbert J, Tamplin A, Secher SM, Pearson J. Short-term outcome of major depression: II. Life events, family dysfunction, and friendship difficulties as predictors of persistent disorder. J Am Acad Child Adolesc Psychiatry 1997; 36: 474-80.

13 Parker G, Tupling H, Brown LB. A Parental Bonding Instrument. Br J Med Psychol 1979; 52: 1-10.

14 Craig $C L$, Marshall AL, Sjöström M, Bauman AE, Booth ML, Ainsworth $B E$, et al. International physical activity questionnaire: 12 country reliability and validity. Med Sci Sports Exercise 2003; 35: 1381-95.

15 Pearson TA, Mensah GA, Alexander RW, Anderson JL, Cannon RO, Criqui M et al. Markers of inflammation and cardiovascular disease: application to clinical and public health practice: a statement for healthcare professionals from the centers for disease control and prevention and the American Heart Association. Circulation 2003; 107: 499-511.

16 Wallace TM, Levy JC, Matthews DR. Use and abuse of HOMA modelling. Diabetes Care 2004; 27: 1487-95.

17 Yasmin, Brown MJ. Similarities and differences between augmentation index and pulse wave velocity in the assessment of arterial stiffness. QJM 1999; 92: 595-600.

18 Mattace-Raso FUS, van der Cammen TJM, Hofman A, van Popele NM, Bos $\mathrm{ML}$, Schalekamp MA, et al. Arterial stiffness and risk of coronary heart disease and stroke: the Rotterdam Study. Circulation 2006; 113: 657-63.

19 Golden SH, Lazo M, Carnethon M, Beroni AG, Schreiner PJ, Diez Roux AV, et al. Examining a bidirectional association between depressive symptoms and diabetes. JAMA 2008; 299: 2751-9.

20 Lustman PJ, Clouse RE. Depression in diabetes: the chicken or the egg? Psychosom Med 2007; 69: 297-9.

21 Pearson S, Schmidt M, Patton G, Dwyer T, Blizzard L, Otahal P, et al. Depression and insulin resistance: cross-sectional associations in young adults. Diabetes Care 2012; 33: 1128-33.

22 Meng L, Chen D, Yang Y, Zheng Y, Hui R. Depression increases the risk of hypertension incidence: A meta-analysis of prospective cohort studies. $J$ Hypertens 2012; 30: 842-51.

23 Dong JY, Zhang YH, Tong J, Qin LQ. Depression and risk of stroke: a meta-analysis of prospective studies. Stroke 2012; 43: 32-7.

24 Williams B, Lacy PS. Central haemodynamics and clinical outcomes: going beyond brachial blood pressure? Eur Heart J 2010; 31: 1819-22.

25 Caspi A, Harrington $H$, Moffitt TE, Milne BJ, Poulton R. Socially isolated children 20 years later: risk of cardiovascular disease. Arch Paediatrics 2006; 8: 805-11. 
26 Danese A, Moffitt TE, Harrington H, Milne BJ, Polanczyk G, Pariante CM, et al. Adverse childhood experiences and adult risk factors for age-related disease: depression, inflammation and clustering of metabolic risk markers. Arch Paed Adolesc Med 2009; 163: 1135-43.

27 Danese A, Moffitt TE, Pariante CM, Ambler A, Poulton R, Caspi A. Elevated inflammation levels in depressed adults with a history of childhood maltreatment. Arch Gen Psychiatry 2008; 65: 409-16.

28 Vreeburg SA, Hartman CA, Hoogendijk WJG, van Dyck R, Zitman FG, Ormel J, et al. Parental history of depression or anxiety and the cortisol awakening response. Br J Psychiatry 2010; 197: 180-5.
29 Cohen-Woods S, Craig IW, McGuffin P. The current state of play on the molecular genetics of depression. Psychol Med 2013; 43: 673-87.

30 Andreassen OA, Djurovic S, Thompson WK, Schork AJ, Kendler KS, O'Donovan MC, et al. Improved detection of common variants associated with schizophrenia by leveraging pleiotropy with cardiovascular-disease risk factors. Am J Hum Gen 2013; 92: 197-209.

31 Ryan JP, Sheu LK, Critchley HD, Gianaros PJ. A neural circuitry linking insulin resistance to depressed mood. Psychosomatic Med 2012; 74: 476-82.

32 Kubzansky LD, Adler GK. Aldosterone: a forgotten mediator of the relationship between psychological stress and heart disease. Neurosci Biobehav Rev 2010; 34: 80-6.

\section{Serotonin}

Philip J. Cowen

Serotonin is a neurotransmitter conserved through at least 500 million years of nervous system evolution. Serotonin orchestrates adaptive responses to aversive stimuli in invertebrates and an analogous role can be discerned in the more complex behavioural repertoire displayed by mammals to adversity. However, this formulation fails to capture the range of human social behaviours influenced by serotonin, for example, affiliation, empathy and cooperation. In a psychopharmacology experiment I received paroxetine for three weeks. This boost in brain serotonin levels failed to alter my subjective ratings of mood and anxiety. My wife felt differently; 'Can't you stay on it?', she said. 